\title{
Characterization of Thin Film Composite Membranes Using Porosimetry and X- Ray Microscopy
}

\author{
Seetha S Manickam ${ }^{1}$, Jeff Gelb ${ }^{2}$, Jeffrey R. McCutcheon ${ }^{1}$ \\ ${ }^{1}$ Department of Chemical, Materials \& Biomolecular Engineering, University of Connecticut, Storrs, \\ CT, USA \\ ${ }^{2}$ Xradia Inc., Pleasanton, CA, USA
}

Engineered osmosis is a membrane-based technology employing osmotic pressure gradients to desalinate water (forward osmosis, FO) and produce power (pressure retarded osmosis, PRO). Internal concentration polarization (ICP) is one of the most important phenomena limiting commercialization of EO. Structural characteristics of the membrane greatly influence the ICP phenomena and are expressed using the structural parameter, $\mathrm{S}$ as

$$
S=\frac{t \tau}{\varepsilon}
$$

where, $t$ is the thickness of the support layer in thin film composite (TFC) membranes, $\tau$ is the tortuosity of the support structure and $\varepsilon$ is the porosity of the support layer. Currently, this parameter is only calculated using experimental flux measurements. Characterization techniques for polymeric materials are underdeveloped [1] and thus the role of the membrane structure in ICP is not fully understood. In this work, two TFC reverse osmosis membranes have been adopted to explore use of different characterization techniques. The two membranes, denoted BW30 and SW30-XLE, were obtained from Dow Water and Process Solutions and are traditionally used for reverse osmosis desalination. Two characterization techniques were used in this study; an analytical technique, mercury intrusion porosimetry (MIP) and a non-destructive 3D imaging technique, x-ray microscopy (XRM). Pore diameter distributions and porosities were obtained from MIP from which tortuosity values were calculated using empirical relations. For the imaging technique, a multi-length scale XRM approach was used. Micron-scale imaging with an Xradia MicroXCT enabled calculation of the structural metrics (porosity, tortuosity and thickness) whereas nano-scale imaging with an Xradia UltraXRM-L200 enabled examination of the microstructure in greater detail. From the micron-scale XRM images, porosity distributions as a function of depth were obtained which is very useful information for EO membranes. It is desirable that surface porosity is high for these membranes to maximize the osmotic pressure gradient available across the selective layer. S values was calculated using the structural metrics obtained from both the MIP and XRM techniques and were compared to the back-calculated value obtained from experimental flux measurements. A large deviation was found between the values obtained from the characterization methods and that obtained from the flux measurements due to the incomplete wetting of the hydrophobic polysulfone layer during FO flux tests. Further, comparing the S values obtained from the two characterization techniques enables to leverage the differences in their measurement methods. The results obtained from this study can help understand mass transport through TFC membranes as well as inform future membrane design in order to maximize their performance in EO technologies. 


\section{References:}

[1] S Manickam, S.; McCutcheon, J. R., Journal of Membrane Science 407-408 (2012), p. 108-115.

[2] The authors acknowledge financial support from the National Science Foundation (CBET- 0933553, CBET) and the University of Connecticut Center for Clean Energy Engineering. The authors also wish to thank Dow Water \& Process Solutions for providing the membranes used in this study and Mike Marsh (VSG) for assistance with the image analysis.

\begin{tabular}{|c|c|c|c|c|c|c|}
\hline & \multicolumn{3}{|c|}{ Analytical (MIP) } & \multicolumn{3}{c|}{ Imaging (MicroXCT) } \\
\cline { 2 - 7 } & Porosity, $\varepsilon \%$ & Tortuosity, $\tau$ & Thickness ${ }^{*}, \mathrm{t} \mu \mathrm{m}$ & Porosity, $\varepsilon \%$ & Tortuosity, $\tau$ & Thickness, $\mathrm{t} \mu \mathrm{m}$ \\
\hline BW30 & $26.63 \pm 4.06$ & $1.120 \pm 0.013$ & $148.3 \pm 6.3$ & $34.91 \pm 1.94$ & $1.216 \pm 0.046$ & $142.0 \pm 1.9$ \\
\hline SW30-XLE & $36.20 \pm 5.51$ & $1.634 \pm 0.006$ & $151.7 \pm 2.5$ & $43.49 \pm 1.22$ & $1.315 \pm 0.164$ & $148.7 \pm 3.0$ \\
\hline
\end{tabular}

This measurement was made using a micrometer.

Table 1. Porosity, tortuosity and thickness estimates for BW30 and SW30-XLE from analytical porosimetry and XRM imaging techniques. $\mathrm{n}=3$ and 2 for porosity and tortuosity measurements obtained from analytical and imaging techniques, respectively. $\mathrm{n}=10$ for both thickness measurements.

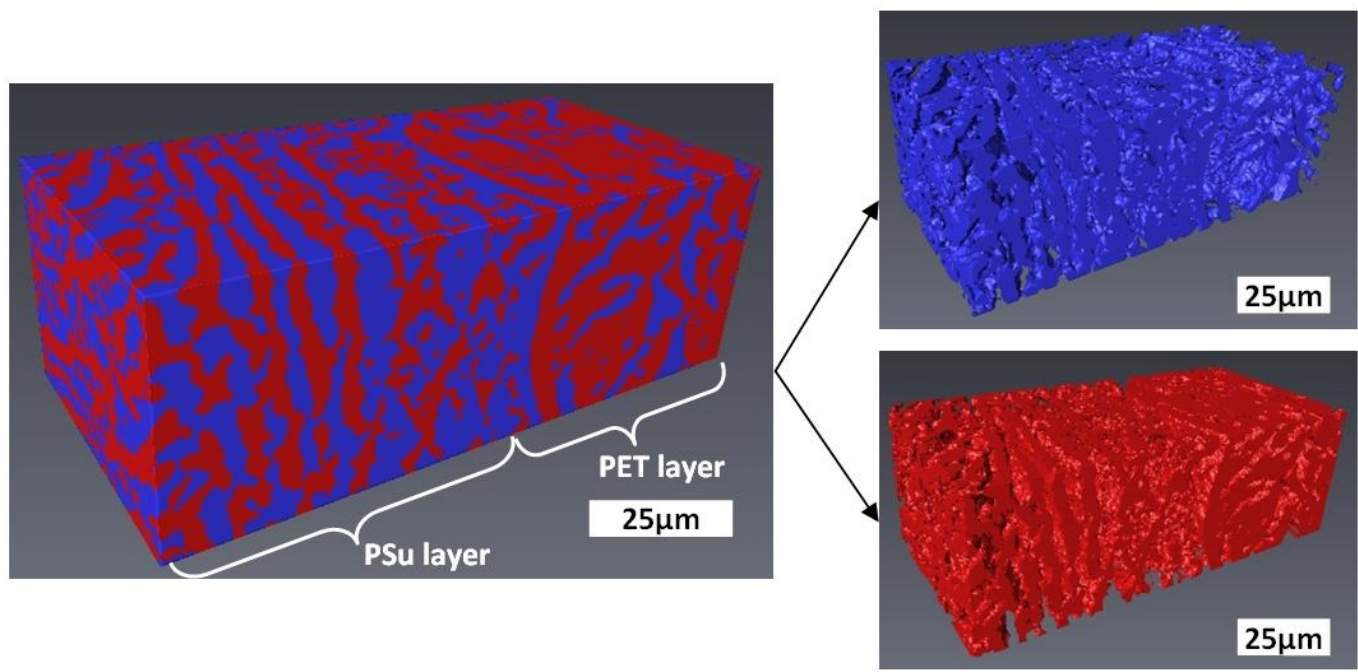

Figure 1. Surface renderings, obtained using Avizo ${ }^{\mathrm{TM}}$ Fire, of the 3D XRM images of SW30-XLE. The image on the left shows the complete structure of the membrane where blue regions denote pore space and red regions denote the polymer matrix. This image can be deconvoluted into pore phase only and polymer matrix only as shown by the images on the right. 